\title{
Reward Prediction Error Signaling during Reinforcement Learning in Social Anxiety Disorder is altered by Social Observation
}

\author{
Michael P. I. Becker ${ }^{1}$, Rolf Voegler ${ }^{1}$, Jutta Peterburs ${ }^{1,2}$, David Hofmann ${ }^{1}$, Christian Bellebaum ${ }^{2}$, \\ Thomas Straube ${ }^{1}$
}

1 Institute of Medical Psychology and Systems Neuroscience, University of Münster, Von-EsmarchStr. 52, 48149 Münster, Germany

2 Department of Biological Psychology, Institute for Experimental Psychology, Heinrich-HeineUniversity, Universitätsstraße 1, 40225 Düsseldorf, Germany

Address for correspondence:

Michael P. I. Becker

Institute of Medical Psychology and Systems Neurosciences

University of Muenster, Germany

Phone: +492518352798

Email: beckermi@uni-muenster.de

Orcid: https://orcid.org/0000-0001-6458-8921

\section{Acknowledgments and Disclosures}

A first draft of this manuscript has been published as a preprint on BioRxiv:

The authors report no biomedical financial interests or potential conflicts of interest. 


\section{ABSTRACT}

BACKGROUND: Dysfunctional expectations of impending social or performance outcomes are core features of Social Anxiety Disorder (SAD) but often lack formal definition in clinical research. Reinforcement learning (RL) models offer a framework to define changes in outcome expectations in a formal way by computing the prediction error (PE). This study quantifies the updating of expectations by PEs in SAD and investigates alterations in $R L$ regions associated with PE signaling.

METHOD: 48 adult participants (24 diagnosed with SAD and 24 age-, gender-, and educationmatched healthy controls $(H C))$ underwent event-related functional magnetic resonance imaging while learning from probabilistic feedback. Crucially, both groups completed two parallel versions of the task: one in which they learned under scrutiny (social observation) and one in which they learned without being overtly evaluated (non-social control condition).

RESULTS: Coupling to prediction errors in SAD was elevated in dorsomedial prefrontal cortex (DMPFC) when learning under observation. These findings provide the first evidence that $P E$ signaling during social performance situations in SAD is associated with hypersensitive response signatures in DMPFC, a brain region associated with using others' value standards as a proxy for one's own value standards. Dynamic Causal Modelling further revealed that RPE-modulated connectivity from ventral pallidum to DMPFC during observation was reduced in $S A D$.

CONCLUSIONS: The present results corroborate a crucial role of DMPFC in SAD, which corresponds to dysfunctional expectations about others' alleged performance standards that play a prominent role in current models of the disorder.

Keywords: feedback learning, functional magnetic resonance imaging (fMRI), reward prediction error (RPE), observation, social anxiety disorder 


\section{Introduction}

Expectations of failure and persistent fear responses during social performance or interactions characterize Social Anxiety Disorder (SAD; American Psychiatric Association, 2013). Patients suffering from SAD most often dread exposure to scrutiny by others, set exaggerated standards for the success of their own social behavior, and invest a lot of time ex post facto to recapitulate negative aspects of past interactions (Rapee \& Heimberg, 1997). Functional models of social anxiety and its clinical manifestation, SAD, converge on the maintaining role of dysfunctional expectations in the development and exacerbation of avoidance and anxiety (Clark \& Wells, 1995; Rapee \& Heimberg, 1997). In particular, dysfunctional expectations about impending social or performance situations are core features of SAD. In general, altered expectations can result from avoidance behaviors and help reduce anxiety (Lovibond et al., 2008). In SAD, situation-specific expectations bias predictions regarding the evaluation of one's actions by others in social situations (Rief et al., 2015, Rapee \& Heimberg, 1997). Hence, negative predictions of one's own performance in social situations supposedly increase the risk of SAD-related avoidance behavior and might even foster maladaptive social strategies that increase the risk of negative evaluation (see reviews by Rief et al., 2015, Hofmann, 2007, Clark \& Wells, 1995). The degree of alteration in these predictions might further affect the course and severity of the disorder as well as responsiveness to therapy (Safren et al., 1997; Delsignore \& Schnyder, 2007; El Alaoui et al., 2015).

The updating of predictions is a mechanism that is hypothesized to be altered in anxiety disorders (see reviews by Grupe \& Nitschke, 2013, Cavanagh \& Shackman, 2015, Paulus \& Stein, 2006, Paulus \& $\mathrm{Yu}, 2012$ ). In particular, altered updating may be characterized by (a) altered prediction errors (White et al., 2017, Homan et al., 2019) and (b) altered learning rates for disorder-relevant stimuli (Browning et al., 2015, Koban et al. 2018, Homan et al., 2019) as formalized by reinforcement learning (RL) models. Specifically in SAD, we would expect excessive prediction errors to performance feedback as well as lower learning rates if the updating of predictions takes place in socially evaluative contexts. Previous research in subclinical anxiety, Post-traumatic Stress Disorder, and SAD has shown that individual differences in learning rates predict individual differences in symptom severity (Browning et al., 2015, Homan et al., 2019, Koban et al., 2017).

RL allows for the investigation of prediction errors on the trial level as well as of overall learning rates but requires a framework of probabilistic learning to do so. Probabilistic learning in SAD has previously been investigated behaviorally (Koban et al., 2017) as well as with respect to its electrophysiological correlates (Voegler et al., 2019). Voegler et al. (2019) found that SAD patients learned better from negative feedback in a control condition and learned worse from negative feedback in a socially evaluative context. In addition, Koban et al. (2017) found that SAD patients did not exhibit the positivity bias present in control subjects and that patients' fear of negative evaluation was negatively associated with learning rates. Moreover, a number of studies have investigated subclinical social anxiety (Abraham \& Herrmann, 2015, Stevens et al., 2014, Piray et al., 2019, Hunter et al., 2019). These studies generally report altered learning with regard to socially evaluative information while presenting mixed findings regarding altered learning rates in individuals with heightened social anxiety. However, subclinical symptom severity is relatively low in these studies and their relevance for clinical manifestation of SAD remains questionable. In sum, a growing body of evidence suggests that feedback learning and updating are biased towards negative information in clinical and non-clinical social anxiety.

Reward Prediction Errors (RPEs) have been shown to be associated with activation of a rewardlearning network (RLN) comprising ventral striatum (VS), lentiform nucleus, ventromedial prefrontal cortex (VMPFC), and midbrain nuclei (Haber \& Behrens, 2015; Pauli et al., 2018). Additionally, RPEs coding for social variables like others' advice (Behrens et al., 2008) or other's value standards (Nicolle 
et al., 2012) are coupled to activation of a network associated with mentalizing of others' goals and intentions (, Wittmann et al., 2016; Schurz et al., 2014), comprising Area 9 of dorsomedial prefrontal cortex (DMPFC) and temporoparietal junction. DMPFC has also been shown to respond to the perception of scrutiny (Gimenez et al., 2012, Becker et al., 2014). In SAD, socially evaluative contexts trigger differential responses in VS (Becker, Simon, et al., 2017) as well as in regions of medial prefrontal cortex (MPFC, Pujol et al., 2013), and alter functional connectivity patterns (Gimenez et al., 2012, Heitmann et al., 2016). In particular, performance feedback given to SAD patients during social observation (Becker et al., 2017) has been linked to these alterations in network activation. Similar findings from healthy subjects with heightened but subclinical social anxiety suggest that MPFC activation is blunted during learning and feedback processing (Peterburs et al., 2016; Piray et al., 2019). However, it is as yet unknown if patients with clinically significant SAD show alterations of RL network activation during prediction error signaling and updating. More importantly, we do not know if RPE-related network activation has a role in triggering biased expectations regarding the evaluation by others in social situations.

A critical aspect of a socially evaluative situation is the immanent feeling of being observed by someone. In order to trigger this feeling, we used an observation manipulation during a probabilistic feedback-learning task (Frank, 2004) to investigate if indices of updating during RL are altered in socially evaluative contexts. Specifically, we expected that updating of learned associations takes place at a slower rate during observation in SAD. Behaviorally, lower learning rates in SAD point towards slower updating in more anxious individuals (Browning et al., 2015). The probabilistic feedback-learning task also allows assessing the differential contributions of positive feedback and negative feedback on learning. Based on our previous research (Voegler et al., 2019), we hypothesized that HC would learn better from positive feedback, while SAD patients would show better learning from negative feedback, especially in the observation condition. On the brain functional level, RPEs during feedback presentation were expected to predict activation of a rewardlearning network comprising ventral striatum (VS), lentiform nucleus, and ventromedial prefrontal cortex (VMPFC) (Haber \& Behrens, 2015; Pauli et al., 2018). We expected observation to modulate RPEs in SAD patients. Analogous to the socio-emotional hypersensitivity they typically exhibit in evaluative social situations and based on findings from several lines of research, we expected SAD patients to show hypersensitive response signatures in medial prefrontal cortex (Voegeler et a., 2019; Bas-Hogendam et al., 2019, Heitmann et al., 2016). Based on these studies' coordinates, we hypothesized an interaction of observation and PEs would recruit Area 9 of DMPFC. We also assumed that activation in VS and lentiform nucleus would show this group-by-observation interaction. Recent studies have shown differences between SAD and HC in VS activation to different feedbacks implying that these differences might indicate blunted signaling of positive Pes (Boehme et al., 2014, Becker et al., 2017). Other studies have shown differences between generalized anxiety disorder and HC in activation of lentiform nucleus, implying that these differences might indicate excessive signaling of negative PEs (White et al., 2017).

As RPEs are a likely candidate mechanism for the modulation of connection strength between regions (den Ouden et al., 2011), another aim of the present study was to assess differences in effective connectivity between SAD and HC. In an earlier study, Heitmann et al. (2016) identified aberrant functional connectivity in both DMPFC and VS in SAD. Based on their findings, we used Dynamic Causal Modelling to model dependencies between the mentalizing network and the reward network. A hierarchical analysis based on Parametric Empirical Bayes (PEB; Friston et al., 2016) allowed us to model and test the following predictions while controlling for between- and withinsubject variances: (1) the RPE modulates the effective connectivity between the mentalizing network and the reward network, (2) observation gates this connectivity, and (3) this gating is amplified in 
bioRxiv preprint doi: httos://doi org/10.1101/821512. this version posted October 30 2019. The copvriaht holder for this preprint (which was not certified by peer review) is the author/funder, who has granted bioRxiv a license to display the preprint in perpetuity. It is made available under aCC-BY-NC-ND 4.0 International license.

SAD. Specifically, we tested a model containing RPEs as modulatory input against a null model that only featured valence (thus disregarding the magnitude information of the RPE). 


\section{Methods and Materials}

\section{Sample characteristics}

Participants were recruited at the Institute of Medical Psychology and Systems Neuroscience at the University of Muenster, Germany. FMRI data from 24 patients with a DSM-IV diagnosis of SAD (mean age: $25.42 \pm 2.78$ years; 14 females) and 24 healthy control subjects (HC) without any diagnosis (mean age: $24.46 \pm 4.17$ years; 16 females) were analyzed. HC were matched to SAD patients with regard to age, gender, and years of education. Before participation, all subjects were screened for neurological and mental disorders. Exclusion criteria for both groups included a history of or currently present psychotic, substance-related, or neurological disorders, in particular severe medical conditions that might influence neurocognitive function (e.g., head injury with loss of consciousness).

The German version of the Structured Clinical Interview (SCID) for DSM-IV (SKID-I; Wittchen et al., 1997) was used to assess patients' current diagnostic status. All interviews were conducted by an experienced clinical psychologist (R.V.). None of the subjects fulfilled the criteria for a diagnosis of a current episode of major depression, obsessive-compulsive disorder, general anxiety disorder, eating disorder, psychotic disorder, or substance abuse as defined by the SCID. Four SAD patients reported previous episodes of major depressive disorder. Six of the SAD patients were in cognitive-behavioral treatment and two received psychopharmacological treatment (both with citalopram). HC and SAD were administered the Beck-Depression Inventory (BDI-II, Beck, et al., 2006), the Social Phobia Scale (SPS), the Social Interaction Anxiety Scale (SIAS, Mattick and Clarke, 1998), and the self-report version of the Liebowitz Social Anxiety Scale (LSAS, Liebowitz, 1987).

Written informed consent was obtained before starting the experimental procedure. The study procedure conforms to the ethical standards of the Declaration of Helsinki and was approved by the Ethics Committee of the German Psychological Society (Deutsche Gesellschaft für Psychologie, DGPS). All subjects received monetary reimbursement for participation, including additional incentives based on learning performance in the experimental task (see next section). All participants, except one, also participated in a separate EEG study (Voegler et al., 2019).

\section{Experimental design}

\section{Observation manipulation}

Before starting the experimental procedure, controls and patients were informed that they would be exposed to two different experimental conditions in two separate task runs. In one condition they would be observed with a video camera that was mounted to the head coil (observation condition), with the video feed being watched by an observer who would critically evaluate their performance. In the other condition, the camera was not mounted and subjects were explicitly informed that there would be no observation (control condition).

After each run, participants were asked to rate their arousal level and feelings of unpleasantness related to positive and negative feedback on Likert scales from 1 (low arousal/not unpleasant at all) to 9 (extreme arousal/extremely unpleasant). After the observational run, this questionnaire included a question that assessed how uncomfortable subjects had felt while being observed, also on a Likert scale ranging from 1 (not uncomfortable at all) to 9 (extremely uncomfortable).

\section{Probabilistic learning task}

The experimental paradigm was an adapted version of the probabilistic learning task described by Frank et al. (2004). Participants learned to associate pairs of Japanese Hiragana characters with monetary wins and losses. We used different sets of stimuli in the two task runs to ensure that 
feedback learning was reset at the beginning of each run, and overall, 12 parallel versions of the task were used. Each version realized a specific mapping of a stimulus pair to a contingency and feedback, for the observation and control conditions respectively. The order of observation and control condition and the assignment of the different versions of the experiment to the conditions were counterbalanced across participants.

On each learning trial, participants were presented with one of three different stimulus pairs $(A B, C D$, EF) composed of two Japanese Hiragana characters, respectively. Participants were asked to choose one of these characters on every trial by pressing the right or left button of an MR-compatible response box. Subjects were informed that their choices determined the monetary outcomes of each trial (win or loss of 20 Eurocent) and therefore the amount of money won at the end of the experiment. Subjects did not receive explicit information regarding the probabilistic nature of the task or the exact reward contingencies. The outcome of each choice was given as symbolic feedback (either triangle or circle, counterbalanced across subjects to wins and losses (see Fig. 1). For stimulus pair $A B$, choosing stimulus $A$ was associated with a win in $80 \%$ of trials, while choosing stimulus $B$ was associated with a win in $20 \%$ of trials. Accordingly, this ratio was $70: 30$ for stimulus pair $C D$, and 60:40 for stimulus pair EF.

[insert Figure 1 about here]

The sequence and time course of stimulus presentation in one trial of the task is depicted in Figure 1. At the beginning of each trial, a fixation cross was presented. Then, one stimulus pair (either $A B, C D$, or EF) was displayed until subjects made a left or right response on the response box (the response was indicated by a colored ring which appeared around the chosen stimulus of the respective pair). If response latency exceeded $3500 \mathrm{~ms}$, subjects were prompted to respond more quickly on future trials and the current trial was aborted. Subsequently, feedback was presented for $1000 \mathrm{~ms}$. The experiment was controlled using Presentation software (Neurobehavioral Systems Inc., Berkeley, CA, USA). Each condition comprised 3 task blocks in each of which each stimulus pair was presented 20 times, thus amounting to a total of 180 trials per run and 360 trials in total.

After each of the two conditions, a transfer of learning (ToL) block assessed the degree to which subjects prioritized learning from wins over learning from losses or vice versa. In these TOL blocks, the symbols A (associated with a win rate of $80 \%$ ) and B (associated with a win rate of $20 \%$ ) were paired with each of the other stimuli $C$ through $F$ (associated, on average, with a win rate of $50 \%$ ) to form 40 new stimulus pairs ( 20 involving A, 20 involving B) that had never before been encountered by the subjects. No feedback was presented during the test blocks. If participants prioritized learning from positive feedback over learning from negative feedback, they should score higher on pairs that included $A$ than on the pairs that included $B$ (positive transfer). If they prioritized learning from negative feedback over learning from positive feedback, they should score higher on pairs that included $B$ than on the pairs that included $A$ (negative transfer). The ratio $A$ (chosen)/B(not chosen) therefore reflects an implicit tendency to learn from positive or negative feedback.

\section{Functional MRI data acquisition and preprocessing}

Data acquisition was carried out with a Siemens 3 Tesla Magnetom PRISMA and a 20-channel Siemens Head Matrix Coil. Structural images were acquired using a sagittal magnetization T1weighted (MPRAGE) sequence ( $T R=2130 \mathrm{~ms}, T E=2.28 \mathrm{~ms}$, voxel size $=1 \mathrm{~mm}$ isotropic, flip angle $=8^{\circ}$ ) with 192 slices. The functional images were collected by using 210 volumes (for each of the six sessions) of a gradient-echo planar sequence sensitive to BOLD contrast ( $T R=2080 \mathrm{~ms}, T E=30 \mathrm{~ms}$, matrix $=92 \times 92$ voxel, FOV $=208 \mathrm{~mm}$, flip angle $\left.=90^{\circ}\right)$. Each volume consisted of 36 axial slices (thickness $=3 \mathrm{~mm}$, gap $=0.3 \mathrm{~mm}$, in-plane resolution $=2.26 \times 2.26 \mathrm{~mm}^{2}$, voxel size $=2.3 \times 2.3 \times 3 \mathrm{~mm}$ ). A 
shimming field was applied before functional imaging commenced to minimize magnetic field inhomogeneity.

All preprocessing steps were carried out using the Data Processing \& Analysis of Brain Imaging (DPABI V3.1_180801, Yan et al., 2016) toolbox, which is based on SPM12 (version 7487). The first 5 data volumes were discarded due to spin saturation effects. The remaining volumes were slice-time corrected and realigned using a six-parameter (rigid body) linear transformation. The anatomical and functional images were co-registered and then segmented into gray matter (GM), white matter (WM), and cerebrospinal fluid (CSF). Functional data were spatially normalized to MNI standard space with DARTEL (Ashburner, 2007) and resampled to $2 \mathrm{~mm}$ isotropic voxels. Finally, the functional data were spatially smoothed with a $6 \mathrm{~mm}$ full width at half maximum (FWHM) Gaussian kernel.

\section{Models and Statistical analyses}

\section{Computational model of probabilistic learning}

We used a standard Q-learning algorithm (Daw, 2009) to generate estimates of Q-values and RPEs from choices and the associated feedback on every trial $t$. For all $t>1$, the model estimates trial-bytrial sequences of six expected values $Q_{A}, Q_{B}, Q_{C}, Q_{D}, Q_{E}$ and $Q_{F}$ for the three stimuli pairs $i=A B, C D$, $E F$ separately in each condition $o \in$ \{observation, control\}. At the start of each condition, all Q-values were initialized to 0 and trials missed due to slow responding were skipped because no gain or loss feedback was presented. For each condition $o$, a trial-by-trial update of $Q$ of the chosen option was implemented according to the following equation:

$$
Q_{\text {chosen|o }}(t+1)=Q_{\text {chosen|o }}(t)+\alpha_{i \mid o} * P E_{i \mid o}(t),
$$

where $\alpha_{\mathrm{i} \text { o。 }}$ represents the learning rate of the respective pair i given the observation condition $\mathrm{o}$, and $\mathrm{PE}_{\mathrm{i} \mid \mathrm{o}}$ represents the prediction error of the respective pair $\mathrm{i}$ given the observation condition $\mathrm{o}$ according to

$$
P E_{i \mid 0}(t)=R_{\text {chosen|o }}(t)-Q_{\text {chosen } \mid o}(t)
$$

$\mathrm{R}_{\text {chosen }} \mathrm{o}(\mathrm{t})$ represents the presented outcome associated with the choice in that particular trial $\mathrm{t}$ : either +1 in case of a win or -1 in case of a loss.

The probability of the individual's actual choice on each trial $t$ was estimated based on the softmax rule for each pair i:

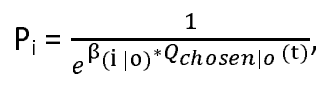

where $\beta_{(\mathrm{i} \mid \mathrm{o}}$ represents the inverse temperature controlling the degree of exploration inherent in choices.

\section{General Linear Model}

First-level single-subject analyses

Based on our hypothesis that indicators of updating during $\mathrm{RL}$ are altered in socially evaluative contexts in SAD patients, we set up a general linear model (GLM) with parametric modulators. This GLM accounted for 3 phases in every trial: (1) presentation of a stimulus pair, (2) participants' choices, and (3) feedback presentation. A vector of onsets convolved with a canonical hemodynamic response function (hrf) represented each phase, respectively. Additionally, the hrf assigned to the feedback phase was parameterized by PE values stemming from RL modelling (see Computational 
model of probabilistic learning). In order to quantify differences related to observation conditions, single-subject beta-weights for these 4 regressors were estimated separately for the observation condition and the control condition, resulting in 8 task-related regressors. Additionally, we included 6 head motion parameters in the GLM.

Second-level random-effects group analyses

Second-level analyses were performed using permutation testing (10?000 permutations) in PALM (Winkler et al., 2014). First, contrasts were defined to test for main effects of RPE as well as interactions with diagnosis (SAD, HC) and interactions with condition (observation, control) and diagnosis. Based on these contrasts, t-maps were computed from subjects' first level beta estimates, and Threshold-free Cluster Enhancement (TFCE) maps were derived as implemented in PALM. We used masks from the Brainnetome Atlas (A9m; http://atlas.brainnetome.org/bnatlas.html) and Reinforcement Learning Atlas (Pauli et al., 2018) and applied FWER-correction of $p$-values $(\alpha=0.05)$.

\section{Dynamic Causal Modelling}

We used Dynamic Causal Modelling (DCM) (Friston et al., 2003; Stephan et al., 2010) as implemented in SPM12 (r6591) to investigate effective connectivity. Subject-specific time series were extracted from regions of interest that were selected on the basis of the second-level random-effects (RFX) analysis. Time series were extracted from the clusters in Area 9 and VS identified by the significant interaction of RPE and diagnosis. The first eigenvariate was then computed across all voxels within these clusters. The resulting time series were adjusted for effects of no interest by specifying an Fcontrast that only included the feedback condition and its parametric modulator. A bilinear one-state DCM model was constructed assuming recursive connections between both regions of interest. All feedback trials were used as driving inputs on both regions of interest. RPE parametric modulators were used as modulating inputs on the recursive connections between Area 9 and VS. DCMs were fitted to the data for observation and control conditions in both groups. To account for betweensubject variability, DCMs were then subjected to a 3-level hierarchical analysis based on Parametric Empirical Bayes (PEB; Zeidman et al., 2019a, 2019b, Friston et al., 2016). The first level comprised a total of 8 DCM parameters ( 4 for the baseline connections (A-matrix) and 4 for the modulatory connections (B-matrix)) for every subject and observation condition which were subjected to PEB on the second and third levels, respectively. The second level comprised between-subjects effects $\boldsymbol{X}_{\boldsymbol{b}}$, and the third level comprised within-subjects effects $\boldsymbol{X}_{\boldsymbol{w}}$ (Zeidman et al., 2019b). Note that this approach accounts for differences in between-subject variability. In addition, we used a Bayesian model reduction to identify the best model given the data. The procedure derives the model evidence (free energy) by removing one or more connectivity parameters from the full PEB grouplevel model to produce reduced forms of the full model. With this approach, it is possible to obtain evidence for reduced models (and their respective parameters) directly from the fully-connected model and thus provides an efficient search of the model space by scoring each reduced model based on its model-evidence (for details see Friston et al., 2016). The models that maximize model evidence are then selected. After model reduction, we used Bayesian model averaging to average the connectivity parameters of the best models weighted by their evidence. That is, the most probable model will contribute the most to the average. In the result section, we report the parameter estimates of this average over the best models based on a posterior probability threshold >.99. 


\section{Results}

\section{Choice data}

In order to analyze participants' choices during learning, a 3 (pair: $A B, C D, E F$ ) $x 3$ (block: $1^{\text {st }}, 2^{\text {nd }}, 3^{\text {rd }}$ ) $x$ 2 (condition: observation, control) x 2 (group: SAD, $\mathrm{HC}$ ) repeated-measures analysis of variance (rmANOVA) was conducted, with Greenhouse-Geisser-correction applied whenever appropriate. The ANOVA yielded a significant main effect of pair $(F[1.69,77.78]=7.137, p<.05)$ and an interaction of pair $x$ block $(F[3.33,153.12]=8.735, p<.05)$ but no other main effects or interactions (all $F<2.046)$. Mean percentages of correct choices according to condition and pair for SAD patients and HC are shown in Supplementary Figure 1. Within-subjects contrasts revealed significant linear trends of pair $(F[1.00,46.00]=9.37, p<.05)$ as well as the interaction of pair $x$ block $(F[1.00,46.00]=13.13, p<$ .05). As no interactions with group were significant, RL from value comparisons per se seemed to be unimpaired in patients.

\section{Differential contributions of positive and negative feedback to learning}

The TOL blocks showed (descriptive data provided in Figure 2) a 2 (feedback valence: positive, negative) $\times 2$ (condition: observation, control) $\times 2$ (group: SAD, HC) rmANOVA yielded a significant main effect of feedback valence $(F[1.00,46.00]=7.959, p<.05)$ and a significant 3-way interaction of feedback valence, group, and condition $(F[1.00,46.00]=6.456, p<.05)$. Post-hoc t-tests showed that $\mathrm{HC}$ learned better from negative feedback under observation (positive transfer: $M(S E)=65.10 \%( \pm$ $4.90 \%)$; negative transfer: $M(S E)=78.99 \%( \pm 3.05 \%) ; t(23)=-2.88, p<.05)$, while SAD showed reduced learning from negative feedback under observation (positive transfer: $M(S E)=61.63 \%$ ( \pm $5.44 \%)$; negative transfer: $M(S E)=70.83 \%( \pm 4.75 \%) ; t(23)=-2.88, p<.05)$.

[insert Figure 2 about here]

\section{Rating data}

Under observation, SAD also reported more subjective discomfort about receiving negative feedback $(\mathrm{M}(\mathrm{SE})=5.88( \pm .45) ; \mathrm{t}(23)=-2.64, \mathrm{p}<.05)$ and higher arousal levels $(\mathrm{M}(\mathrm{SE})=5.08( \pm .45) ; \mathrm{t}(23)=-$ $2.40, p<.05)$ than $\mathrm{HC}$ (discomfort: $M(S E)=4.38( \pm .35)$; arousal: $M(S E)=3.67( \pm .39)$ ).

\section{Learning rates}

Finally, a regression analysis with learning rates onto LSAS scores was conducted (2 independent variables: (1) learning rates from the observation condition, (2) learning rates from the control condition). As expected, LSAS scores were predicted by learning rates from the observation condition $\left(\beta=-.257, p_{\text {onesided }}<.05\right)$ but not by learning rates from the control condition $(\beta=-.064, p=.67)$. The negative association between learning rates and symptom severity suggests slower updating of learned associations in socially anxious individuals (Figure 3).

[insert Figure 3 about here] 


\section{fMRI}

\section{RPE-related activation}

First, we tested if our model based on RPE parametric modulators for feedback events replicated the RL network (Pauli et al., 2018) across groups and observation conditions. A main effect of RPEweighted feedback-related activation was found in the typical RL network comprising nucleus accumbens, lentiform nucleus (Putamen and Globus Pallidus), VMPFC, amygdala, lateral OFC, and other regions (all maps are FWE-corrected after PALM permutation analysis, $p<.05$ ). Analogous to behavioral choice data, RPE-related activation in VMPFC did not show significant effects of group or observation condition underpinning the assumption that value comparisons per se seemed to be unimpaired in SAD (see Supplementary Figure 2).

\section{Differences in RPE-related activation between groups}

Next, we looked for group differences in RPE-related activation. Activity of a region in DMPFC corresponding to Area 9 selectively tracked reward prediction errors in SAD (peak: $x, y, z=-4,32,36$ ). Figure 4 shows the cluster in Area 9 of DMPFC and the corresponding beta maps (averaged over all voxels within that cluster). Importantly, this same region tracked RPE in SAD preferentially when learning under observation, i.e. betas were higher in the observation than in the control condition (Fig. 5; all maps are FWE-corrected maps after PALM permutation analysis, masks used are from Reinforcement Learning Atlas and Brainnetome Atlas). Correspondingly, a subdivision of the lentiform nucleus also showed stronger coupling to RPE in SAD than in HC (peak: $x, y, z=12,8,-2$; Fig. 5). Furthermore, a cluster in VS showed an interaction of RPE and group with higher activation in SAD than in HC (peak: $x, y, z=-10,9,-2$ ). The interactions in A9 and VP held after controlling for medication status as qualified by an ANCOVA with medication status as covariate (A9: $F[1.00,45.00]=.641, p=$ .43 , VP: F[1.00, 45.00] $=.003, p=.96$ ). No other RL-relevant regions (Pauli et al., 2018) showed significant interactions of RPE, observation and diagnosis and no region showed higher activation for RPE in HC.

[insert Figure 4 about here] 


\section{Session-related effects}

We also asked whether learning effects modulated RPE-related activation differences between the groups. Therefore, we separately modelled predictors for each of the six learning blocks (three for the observation condition and three for the control condition). At the threshold of the other contrasts, no block-related effects were detectable.

\section{Dynamic Causal Modelling}

As RPEs are a likely candidate mechanism for the modulation of connection strength between regions (den Ouden et al., 2011), we used Dynamic Causal Modelling (DCM) to investigate if this mechanism showed differences between SAD and HC under observation. We specified and compared two linear models (see Figure 5): (1) we investigated if RPE signaling, comprising valence and magnitude information, alters connectivity from VS to Area 9 in SAD under observation, (2) we investigated if connectivity from VS to Area 9 was altered by positive and negative feedback (without taking into consideration the magnitude of the RPE) in SAD under observation. Model comparison was based on the Log Bayes Factor (LBF) of the difference in Free Energy of the PEB models and expressed as each model's posterior probability. A better model fit for Model 1 (using RPE as modulatory input) over Model 2 (using only positive and negative Feedback as modulatory input) would suggest that connectivity between VS and A9 in SAD under observation is not only gated by the valence of feedback but also by magnitude information provided by our RL model. Model comparison showed strong evidence in favor of Model 1 over Model 2 (LBF = 7.75), i.e. the magnitude model best explained the data. Under observation, connectivity from VS to A9 in patients did indeed decrease as a function of RPE, i.e., positive RPEs reduced the directed connection from VS to $A 9$ during feedback presentation while negative RPEs increased this connectivity.

[insert Figure 5 about here]

\section{Discussion}

The present study was designed to investigate disorder-related alterations of RL in SAD brought about by social scrutiny. Therefore, patients with a DSM-IV diagnosis of SAD and HC completed a probabilistic feedback learning task (Frank et al., 2004, Voegler et al., 2019) in which they were exposed to social observation and a non-social control condition. Observation led to significantly more reported subjective discomfort in SAD than in $H C$, attesting to the disorder-relevance of the manipulation. Behaviorally, overall learning performance was comparable between SAD and HC, suggesting that SAD were not impaired by deficits in RL per se. However, learning rates in the observation condition predicted severity of social anxiety symptoms. Behaviorally, lower learning rates in more anxious individuals pointed towards slower updating in individuals with higher symptom burden (Browning et al., 2015, Koban et al., 2018). Assessment of TOL revealed that SAD showed a bias for negative learning under observation and in the control condition, while HC only showed a bias for negative learning under observation. This result is in line with the pattern found by Voegler et al. (2019) in an EEG study and Abraham \& Hermann (2015) in a behavioral study.

On the brain functional level, RPEs during feedback presentation were expected to predict activation of a RL-network comprising ventral striatum (VS), lentiform nucleus, and VMPFC (Haber \& Behrens, 2015; Pauli et al., 2018). We found comparable activation in VMPFC to RPE in both groups and both conditions. This suggests that VMPFC did not distinguish between the observation and control conditions (see also Simon et al., 2014, Becker, Simon et al., 2017). Furthermore, VMPFC activation did not distinguish between SAD and HC. Since, the percentage of correct choices was comparable in both groups across the three different contingencies $A B, C D$ and $E F$, and given that VMPFC has been implicated in the coding of value comparisons, this result is in line with our previous suggestion that 
value computations in SAD are functionally similar to those found in control subjects. Hence, we would not assume that VMPFC contributes directly to the differences in TOL.

Based on our hypotheses, we expected SAD patients and controls to represent RPEs differently during observation in VS and Area 9 of the mentalizing network (Wittman et al., 2016, Kanske et al., 2015). In line with this prediction, we found an interaction of observation and RPE, with RPE predicting activation in this region better during observation. Finally, as expected, we found this interaction to be more pronounced in SAD, i.e., RPE in SAD during observation predicted activation in Area 9 significantly better.

The medial part of Area 9 (Fan et al., 2013, Petrides \& Pandya, 1999, Sallet et al., 2013) is located in the DMPFC which is arguably the most important brain region in social inference (Amodio \& Frith, 2006) and has been ascribed a particular role when choices are being made based on the alleged value standards of others in their absence (Hampton et al., 2008, Nicolle et al., 2012, see Joiner et al. for review). Area 9 has been identified as a functional subregion of DMPFC in human resting state (Li et al., 2011) and task FMRI where it is activated when confusing other's performance with ones's own (Wittmann et al., 2016). Our results suggest stronger coupling of Area 9 to RPEs in SAD than in $\mathrm{HC}$, with this coupling further amplified by observation. As being observed increased subjective discomfort in SAD, it is interesting to ask if this discomfort was related to thinking about the expectations of the observers. Area 9 has previously been found to track expectations about the performance of others and to integrate self-related information into these expectations (Wittmann et al., 2016, see Leo \& Seo, 2016 for review). This is consistent with the idea that this region is engaged when mentalizing about a dissimilar other (Mitchell et al., 2006) and learning about the social value of another player during interactions based on reliably reading others strategies (Behrens et al., 2008), imagining others' thoughts and intentions (Saxe \& Powell, 2006), or representing others' personality traits (Van Overwalle, 2009). Nicolle et al. (2014) reported DMPFC activation not only when simulating the established value standards of another person, but also when using one's own value standards as a proxy for the unknown value standards of others. Hence, the computational mechanisms identified by this study are in line with the cognitive mechanism central to the model of the generation and maintenance of anxiety in social/evaluative situations proposed by Rapee \& Heimberg (1997). This model assumes that, in SAD, dysfunctional cognitive mechanisms during observation are characterized by biased predictions regarding the evaluation of one's actions by others in social situations. These biases arise from dysfunctional expectations and possibly result in excessive feedback prediction error signaling. Importantly, previous studies have shown that DMPFC is a region that carries these social PEs (Behrens et al., 2008; Nicolle et al., 2012).

Prior evidence from $\mathrm{FMRI}$ in SAD and subclinical anxiety has implied aberrant DMPFC activation in SAD but has never shown a connection to PE signalling: Sripada et al. (2009) reported altered activation of DMPFC in SAD, albeit reduced during interactions with humans relative to a computer. In subclinical social anxiety, Peterburs et al. (2016) also found reduced activation of MPFC in HSA. However, Heitmann et al. (2014) found elevated anticipatory DMPFC activation in subclinical social anxiety to positive and negative feedback provided by human raters. Becker, Simon et al., (2017) reported a region in MPFC to show an interaction of feedback valence, observation, and SAD diagnosis. However, no study thus far has shed light on the computational mechanisms at the heart of these phenomena. The present study shows for the first time in SAD that RPEs during scrutiny can explain aberrant BOLD responses in DMPFC.

Having established this connection, the next question we asked was whether aberrant BOLD patterns in DMPFC in SAD were the source or the target of aberrant cross-talk with other regions of the RL network. VS has an important role in this network (Haber \& Behrens, 2015). As VS also showed enhanced coupling to RPES in SAD, we aimed to further elucidate the dependencies between both 
network regions by DCM. RPEs have been discussed as a candidate mechanism for the modulation of connection strength between regions (den Ouden et al., 2011, Stephan et al., 2010). We set up a bilinear one-state DCM using RPE as modulating input and used an hierarchical estimation procedure based on PEB to estimate its parameters. This allowed us to estimate interactions of diagnosis and observation in effective connectivity while controlling for within- and between-subject variance. The results clearly favored effective connectivity from VS to A9 modulated by RPE as the best solution over all other modulatory connections. Most importantly, the hierarchical DCM clearly showed that RPE-modulated connectivity from VS to A9 was most pronounced in SAD under observation. We tested a model containing RPE as modulatory input against a null model that only featured valence (thus disregarding the magnitude information of the RPE). This was done to ensure that the RPE was indeed a better predictor than feedback valence alone. Comparison of the FE of these models revealed that RPE magnitude significantly contributed to the explanatory power of the model. As FE punishes the number of model parameters, we made sure to keep the parameter input to both models identical in number.

Earlier studies have reported aberrant functional connectivity (FC) in SAD in DMPFC. Heitmann et al. (2016) identified hyperconnectivity of globus pallidus with DMPFC during presentation of disorderrelated scenes. Gimenez et al. (2014) also reported differences in FC between SAD and HC in regions of medial frontal cortex. However, no study to date has used measures of effective connectivity to establish the directionality of aberrant regional cross-talk in SAD during probabilistic learning. Interestingly, using a very similar probabilistic learning task, Klein et al. (2007) found FC between MPFC and VS to differ for two polymorphisms of the dopamine D2 receptor gene DRD2. However, an important limitation of FC analysis is that it is based on correlational metrics and thus cannot establish the direction of connectivity (Friston, 2011). In other words, measures of FC are not able to infer true coupling, because they map consequences to causes based on statistical dependencies (Friston, 2011). For example, two regions can show substantial FC despite the absence of any true connection, just because of a common input from a third region (Friston, 2011). Moreover, since correlations depend on the level of observation noise, changes in FC arise by merely varying the signal-to-noise ratio, e.g., by increasing the number of time points or the sample size (Friston, 2011). Finally, changes in FC also arise by changing the amplitudes of neuronal fluctuations (Friston, 2011). Metrics of EC offer a way to avoid these pitfalls in network connectivity analysis. Therefore, we used DCM together with PEB to model network dependencies and their possible alteration in SAD. Other than tests based on classical statistics, PEB uses the full posterior density over the connectivity parameters from each subject's DCM to inform results on the group-level. It thus takes into account both the expected strength of the connection and its uncertainty (posterior covariance). In other words, subjects are weighted by the precision of their estimates, such that subjects with noisy estimates contribute less to the group result. We used a hierarchical procedure with three levels to estimate the DCM parameters. We modelled baseline as well as modulatory connections between VS and MPFC on the first level, group differences between SAD and HC on the second level, and differences between the observation and control condition on the third level. This allowed us to assess interactions of diagnosis and observation in the connectivity patterns from VS to A9 at baseline and when modulated by RPE. Hence, our analysis validates the basic findings of earlier studies using FC while surmounting their main limitation in showing a clear directionality in connectivity from VS to A9.

Nonetheless, when interpreting the findings of the present study, other limitations should be kept in mind. The first limitation is the number of 24 participants in each group. However, power calculations based on published RL studies of psychiatric disorders suggest that 24 participants in each group are sufficient to estimate between-group effects of RPE in DMPFC, ACC, and VS (Cremers et al., 2012; Peterburs et al., 2016; Becker, Simon et al., 2017; Jarcho et al., 2015). Furthermore, our 
study has the advantage over earlier studies that we only included patients with a clinical manifestation of SAD. Hence, its validity for clinical questions can be expected to be higher. Second, it should be considered that the SAD group comprised two subjects currently treated with antidepressive medication (SSRI) as well as six subjects who received psychotherapy. While about a third of SAD patients can be expected to take SSRIs, it is not entirely clear if this might influence brain activation related to RL. However, only a relatively small number of subjects was medicated, and no direct effects of medication were statistically significant. Furthermore, a previous study using [15,2 O]-PET showed that administration of an SSRI in SAD does not generally alter the neuronal correlates of processing of social stimuli (Faria et al. 2012).

Taken together, the present study exemplifies a promising new approach to anxiety research by elucidating the role of prediction errors in SAD during social observation. A key finding is the aberrant coding of RPEs during observation in Area 9 of DMPFC - a region previously reported when using one's own value standards as a proxy for others decisions. Current models of SAD assume this as a key mechanism in the maintenance of the disorder (Rapee \& Heimberg, 1997). Hence our findings point toward altered RPE coding in Area 9 as a candidate mechanism for dysfunctional updating of expectations in SAD under observation. Moreover, we were able to show that EC between ventral pallidum and Area 9 was selectively upregulated by RPE in patients only during observation. This finding may contribute to the refinement of diagnostic criteria and differential diagnosis in anxiety disorders and might lead to a deeper understanding of the biological roots of dysfunctional expectations. Furthermore, the combination of observation manipulation by camera and computational modelling might offer a new approach for theranostics, which uses the decoupling of PEs and DMPFC activation under observation as a marker for therapeutic progress. 


\section{References}

Abraham, A., \& Hermann, C. (2015). Biases in probabilistic category learning in relation to social anxiety. Front Psychol, 6, 1218. doi:10.3389/fpsyg.2015.01218

Amodio, D. M., \& Frith, C. D. (2006). Meeting of minds: the medial frontal cortex and social cognition. Nat Rev Neurosci, 7(4), 268-277. doi:10.1038/nrn1884

Arioli, M., Perani, D., Cappa, S., Proverbio, A. M., Zani, A., Falini, A., \& Canessa, N. (2018). Affective and cooperative social interactions modulate effective connectivity within and between the mirror and mentalizing systems. Human Brain Mapping, 39(3), 1412-1427. doi:10.1002/hbm. 23930

Ashburner, J. (2007). A fast diffeomorphic image registration algorithm. Neuroimage, 38(1), 95-113. doi:10.1016/j.neuroimage.2007.07.007

Balsters, J. H., Apps, M. A., Bolis, D., Lehner, R., Gallagher, L., \& Wenderoth, N. (2017). Disrupted prediction errors index social deficits in autism spectrum disorder. Brain, 140(1), 235-246. doi:10.1093/brain/aww287

Bas-Hoogendam, J. M., van Steenbergen, H., Tissier, R. L. M., van der Wee, N. J. A., \& Westenberg, P. M. (2019). Altered Neurobiological Processing of Unintentional Social Norm Violations: A Multiplex, Multigenerational Functional Magnetic Resonance Imaging Study on Social Anxiety Endophenotypes. Biol Psychiatry, doi:10.1016/j.bpsc.2019.03.003

Becker, M. P. I., Simon, D., Miltner, W. H. R., \& Straube, T. (2017). Altered activation of the ventral striatum under performance-related observation in social anxiety disorder. Psychological Medicine, 47(14), 2502-2512. doi:10.1017/S0033291717001076

Boehme, S., Mohr, A., Becker, M. P., Miltner, W. H., \& Straube, T. (2014). Area-dependent time courses of brain activation during video-induced symptom provocation in social anxiety disorder. Biol Mood Anxiety Disord, 4(6). doi:10.1186/2045-5380-4-6

Browning, M., Behrens, T. E., Jocham, G., O'Reilly, J. X., \& Bishop, S. J. (2015). Anxious individuals have difficulty learning the causal statistics of aversive environments. Nature Neuroscience, 18(4), 590-596. doi:10.1038/nn.3961

Button, K. S., Browning, M., Munafo, M. R., \& Lewis, G. (2012). Social inference and social anxiety: Evidence of a fear-congruent self-referential learning bias. Journal of Behavior Therapy and Experimental Psychiatry, 43(4), 1082-1087. doi:10.1016/j.jbtep.2012.05.004

Cavanagh, J. F., \& Shackman, A. J. (2015). Frontal midline theta reflects anxiety and cognitive control: Meta-analytic evidence. Journal of Physiology-Paris, 109(1-3), 3-15. doi:10.1016/j.jphysparis.2014.04.003

Cremers, H. R., Veer, I. M., Spinhoven, P., Rombouts, S. A., \& Roelofs, K. (2014). Neural sensitivity to social reward and punishment anticipation in social anxiety disorder. Front Behav Neurosci, 8, 439. doi:10.3389/fnbeh.2014.00439

Daw, N. (2009). Trial-by-trial data analysis using computational models. In M. R. Delgado, E. Phelps, \& T. Robbins (Eds.), Decision Making, Affect, and Learning: Attention and Performance XXIII: Oxford Scholarship Online.

Delsignore, A., \& Schnyder, U. (2007). Control expectancies as predictors of psychotherapy outcome: A systematic review. British Journal of Clinical Psychology, 46, 467-483. doi:10.1348/014466507X226953

den Ouden, H. E. M., Daunizeau, J., Roiser, J., Friston, K. J., \& Stephan, K. E. (2010). Striatal Prediction Error Modulates Cortical Coupling. Journal of Neuroscience, 30(9), 3210-3219. doi:10.1523/Jneurosci.4458-09.2010

El Alaoui, S., Ljotsson, B., Hedman, E., Kaldo, V., Andersson, E., Ruck, C., ... Lindefors, N. (2015). Predictors of Symptomatic Change and Adherence in Internet-Based Cognitive Behaviour Therapy for Social Anxiety Disorder in Routine Psychiatric Care. PLoS One, 10(4), e0124258. doi:10.1371/journal.pone. 0124258

Fadul, L. C., da Silva, M. T., de Freitas, L. A. R., \& Athanazio, D. A. (2016). Assessment of the circumferential margins, extraprostatic extension and Gleason score in radical prostatectomy specimens: Comparison of a partial embedding method with supplemental total inclusion of 
peripheral tissues. Pathology Research and Practice, 212(3), 217-221.

doi:10.1016/j.prp.2015.12.014

Fan, L., Li, H., Zhuo, J., Zhang, Y., Wang, J., Chen, L., ... Jiang, T. (2016). The Human Brainnetome Atlas: A New Brain Atlas Based on Connectional Architecture. Cereb Cortex, 26(8), 3508-3526. doi:10.1093/cercor/bhw157

Faria, V., Appel, L., Ahs, F., Linnman, C., Pissiota, A., Frans, O., . . Furmark, T. (2012). Amygdala subregions tied to SSRI and placebo response in patients with social anxiety disorder. Neuropsychopharmacology, 37(10), 2222-2232. doi:10.1038/npp.2012.72

Frank, M. J., Seeberger, L. C., \& O'Reilly R, C. (2004). By carrot or by stick: cognitive reinforcement learning in parkinsonism. Science, 306(5703), 1940-1943. doi:10.1126/science.1102941

Friston, K. J., Harrison, L., \& Penny, W. (2003). Dynamic causal modelling. Neuroimage, 19(4), 12731302.

Friston, K., 2011. Functional and effective connectivity: a review. Brain Connect. 1, 13-36. https://doi.org/10.1089/brain.2011.0008

Friston, K. J., Litvak, V., Oswal, A., Razi, A., Stephan, K. E., van Wijk, B. C. M., . . Zeidman, P. (2016). Bayesian model reduction and empirical Bayes for group (DCM) studies. Neuroimage, 128, 413-431. doi:10.1016/j.neuroimage.2015.11.015

Gibbons, F. X., \& Buunk, B. P. (1999). Individual differences in social comparison: development of a scale of social comparison orientation. J Pers Soc Psychol, 76(1), 129-142.

Gimenez, M., Pujol, J., Ortiz, H., Soriano-Mas, C., Lopez-Sola, M., Farre, M., . . Martin-Santos, R. (2012). Altered brain functional connectivity in relation to perception of scrutiny in social anxiety disorder. Psychiatry Research-Neuroimaging, 202(3), 214-223. doi:10.1016/j.pscychresns.2011.10.008

Glascher, J., Daw, N., Dayan, P., \& O'Doherty, J. P. (2010). States versus Rewards: Dissociable Neural Prediction Error Signals Underlying Model-Based and Model-Free Reinforcement Learning. Neuron, 66(4), 585-595. doi:10.1016/j.neuron.2010.04.016

Grupe, D. W., \& Nitschke, J. B. (2013). Uncertainty and anticipation in anxiety: an integrated neurobiological and psychological perspective. Nat Rev Neurosci, 14(7), 488-501. doi:10.1038/nrn3524

Haber, S. N., \& Behrens, T. E. J. (2014). The Neural Network Underlying Incentive-Based Learning: Implications for Interpreting Circuit Disruptions in Psychiatric Disorders. Neuron, 83(5), 10191039. doi:10.1016/j.neuron.2014.08.031

Hampton, A. N., Bossaerts, P., \& O'Doherty, J. P. (2008). Neural correlates of mentalizing-related computations during strategic interactions in humans. Proceedings of the National Academy of Sciences of the United States of America, 105(18), 6741-6746. doi:10.1073/pnas.0711099105

Heitmann, C. Y., Feldker, K., Neumeister, P., Zepp, B. M., Peterburs, J., Zwitserlood, P., \& Straube, T. (2016). Abnormal brain activation and connectivity to standardized disorder-related visual scenes in social anxiety disorder. Human Brain Mapping, 37(4), 1559-1572. doi:10.1002/hbm. 23120

Hermann, C., Ziegler, S., Birbaumer, N., \& Flor, H. (2002). Psychophysiological and subjective indicators of aversive pavlovian conditioning in generalized social phobia. Biological Psychiatry, 52(4), 328-337. doi:Pii S0006-3223(02)01385-9

Homan, P., Levy, I., Feltham, E., Gordon, C., Hu, J., Li, J., . . Schiller, D. (2019b). Neural computations of threat in the aftermath of combat trauma. Nature Neuroscience, 22(3), 470-476. doi:10.1038/s41593-018-0315-x

Hunter, L. E., Meer, E. A., Gillan, C. M. Hsu, M., \& Daw, N. D. (2019). Excessive deliberation in social anxiety. BioRxiv. doi: https://doi.org/10.1101/522433

Jarcho, J. M., Romer, A. L., Shechner, T., Galvan, A., Guyer, A. E., Leibenluft, E., . . Nelson, E. E. (2015). Forgetting the best when predicting the worst: Preliminary observations on neural circuit function in adolescent social anxiety. Dev Cogn Neurosci, 13, 21-31. doi:10.1016/j.den.205.03.002 
Kanske, P., Bockler, A., \& Singer, T. (2017). Models, Mechanisms and Moderators Dissociating Empathy and Theory of Mind. Curr Top Behav Neurosci, 30, 193-206. doi:10.1007/7854_2015_412

Klein, T. A., Neumann, J., Reuter, M., Hennig, J., von Cramon, D. Y., \& Ullsperger, M. (2007). Genetically determined differences in learning from errors. Science, 318(5856), 1642-1645. doi:10.1126/science.1145044

Koban, L., Schneider, R., Ashar, Y. K., Andrews-Hanna, J. R., Landy, L., Moscovitch, D. A., . . Arch, J. J. (2017). Social Anxiety is Characterized by Biased Learning About Performance and the Self. Emotion, 17(8), 1144-1155. doi:10.1037/emo0000296

Lago, T., Davis, A., Grillon, C., \& Ernst, M. (2017). Striatum on the anxiety map: Small detours into adolescence. Brain Research, 1654, 177-184. doi:10.1016/j.brainres.2016.06.006

Lee, D., \& Seo, H. (2016). Neural Basis of Strategic Decision Making. Trends Neurosci, 39(1), 40-48. doi:10.1016/j.tins.2015.11.002

Liebowitz, M. R. (1987). Social phobia. Mod Probl Pharmacopsychiatry, 22, 141-173.

Lovibond, P. F., Saunders, J. C., Weidemann, G., \& Mitchell, C. J. (2008). Evidence for expectancy as a mediator of avoidance and anxiety in a laboratory model of human avoidance learning. Quarterly Journal of Experimental Psychology, 61(8), 1199-1216. doi:10.1080/17470210701503229

Mattick, R. P., \& Clarke, J. C. (1998). Development and validation of measures of social phobia scrutiny fear and social interaction anxiety. Behaviour Research and Therapy, 36(4), 455-470.

Mikita, N., Simonoff, E., Pine, D. S., Goodman, R., Artiges, E., Banaschewski, T., . . Stringaris, A. (2016). Disentangling the autism-anxiety overlap: fMRI of reward processing in a communitybased longitudinal study. Transl Psychiatry, 6(6), e845. doi:10.1038/tp.2016.107

Mitchell, J. P., Macrae, C. N., \& Banaji, M. R. (2006). Dissociable medial prefrontal contributions to judgments of similar and dissimilar others. Neuron, 50(4), 655-663. doi:10.1016/j.neuron.2006.03.040

Moore, A. D. (2015). Method of Attack: A Supplemental Model for Hate Crime Analysis. Indiana Law Journal, 90(4), 1707-1726.

Nakao, T., Sanematsu, H., Yoshiura, T., Togao, O., Murayama, K., Tomita, M., . . Kanba, S. (2011). fMRI of patients with social anxiety disorder during a social situation task. Neurosci Res, 69(1), 67-72. doi:10.1016/j.neures.2010.09.008

Nicolle, A., Klein-Flugge, M. C., Hunt, L. T., Vlaev, I., Dolan, R. J., \& Behrens, T. E. (2012). An agent independent axis for executed and modeled choice in medial prefrontal cortex. Neuron, 75(6), 1114-1121. doi:10.1016/j.neuron.2012.07.023

Park, S. Q., Kahnt, T., Beck, A., Cohen, M. X., Dolan, R. J., Wrase, J., \& Heinz, A. (2010). Prefrontal Cortex Fails to Learn from Reward Prediction Errors in Alcohol Dependence. Journal of Neuroscience, 30(22), 7749-7753. doi:10.1523/Jneurosci.5587-09.2010

Pauli, W. M., Nili, A. N., \& Tyszka, J. M. (2018). A high-resolution probabilistic in vivo atlas of human subcortical brain nuclei. Scientific Data, 5, 180063. doi:10.1038/sdata.2018.63

Paulus, M. P., Hozack, N., Frank, L., \& Brown, G. G. (2002). Error rate and outcome predictability affect neural activation in prefrontal cortex and anterior cingulate during decision-making. Neuroimage, 15(4), 836-846. doi:10.1006/nimg.2001.1031

Paulus, M. P., \& Stein, M. B. (2006). An insular view of anxiety. Biological Psychiatry, 60(4), 383-387. doi:10.1016/j.biopsych.2006.03.042

Paulus, M. P., \& Yu, A. J. (2012). Emotion and decision-making: affect-driven belief systems in anxiety and depression. Trends in Cognitive Sciences, 16(9), 476-483. doi:10.1016/j.tics.2012.07.009

Peterburs, J., Sandrock, C., Miltner, W. H. R., \& Straube, T. (2016). Look who's judging-Feedback source modulates brain activation to performance feedback in social anxiety. Neuroimage, 133, 430-437. doi:10.1016/j.neuroimage.2016.03.036

Petrides, M., \& Pandya, D. N. (1999). Dorsolateral prefrontal cortex: comparative cytoarchitectonic analysis in the human and the macaque brain and corticocortical connection patterns. European Journal of Neuroscience, 11(3), 1011-1036. doi:DOI 10.1046/j.14609568.1999.00518.x 
Piray, P., Ly, V., Roelofs, K., Cools, R., \& Toni, I. (2019). Emotionally Aversive Cues Suppress Neural Systems Underlying Optimal Learning in Socially Anxious Individuals. Journal of Neuroscience, 39(8), 1445-1456. doi:10.1523/Jneurosci.1394-18.2018

Pujol, J., Gimenez, M., Ortiz, H., Soriano-Mas, C., Lopez-Sola, M., Farre, M., . . Martin-Santos, R. (2013). Neural response to the observable self in social anxiety disorder. Psychological Medicine, 43(4), 721-731. doi:10.1017/S0033291712001857

Rapee, R. M., \& Heimberg, R. G. (1997). A cognitive-behavioral model of anxiety in social phobia. Behaviour Research and Therapy, 35(8), 741-756.

Rief, W., Glombiewski, J. A., Gollwitzer, M., Schubo, A., Schwarting, R., \& Thorwart, A. (2015). Expectancies as core features of mental disorders. Current Opinion in Psychiatry, 28(5), 378385. doi: $10.1097 /$ Yco.0000000000000184

Safren, S. A., Heimberg, R. G., \& Juster, H. R. (1997). Clients' expectancies and their relationship to pretreatment symptomatology and outcome of cognitive-behavioral group treatment for social phobia. J Consult Clin Psychol, 65(4), 694-698.

Sallet, J., Mars, R. B., Noonan, M. P., Neubert, F. X., Jbabdi, S., O'Reilly, J. X., . . Rushworth, M. F. (2013). The Organization of Dorsal Frontal Cortex in Humans and Macaques. Journal of Neuroscience, 33(30), 12255-12274. doi:10.1523/Jneurosci.5108-12.2013

Salowe, R. J., O'Keefe, L., Merriam, S., Lee, R., Sankar, P., Miller-Ellis, E. G., .. O'Brien, J. M. (2017). Supplemental Recruitment Methods for the Primary Open-Angle African American Glaucoma Genetics Study. Investigative Ophthalmology \& Visual Science, 58(8).

Schurz, M., Radua, J., Aichhorn, M., Richlan, F., \& Perner, J. (2014). Fractionating theory of mind: A meta-analysis of functional brain imaging studies. Neuroscience and Biobehavioral Reviews, 42, 9-34. doi:10.1016/j. neubiorev.2014.01.009

Sripada, C., Angstadt, M., Liberzon, I., McCabe, K., \& Phan, K. L. (2013). Aberrant Reward Center Response to Partner Reputation during a Social Exchange Game in Generalized Social Phobia. Depression and Anxiety, 30(4), 353-361. doi:10.1002/da.22091

Stephan, K. E., Penny, W. D., Moran, R. J., den Ouden, H. E., Daunizeau, J., \& Friston, K. J. (2010). Ten simple rules for dynamic causal modeling. Neuroimage, 49(4), 3099-3109. doi:10.1016/j.neuroimage.2009.11.015

Stevens, S., Peters, A., Abraham, A., \& Hermann, C. (2014). Enhanced avoidance behavior in social anxiety: Evidence from a probabilistic learning task. Journal of Behavior Therapy and Experimental Psychiatry, 45(1), 39-45. doi:10.1016/j.jbtep.2013.07.007

Swaney, D. L., McAlister, G. C., Wirtala, M., Schwartz, J. C., Syka, J. E. P., \& Coon, J. J. (2007). Supplemental activation method for high-efficiency electron-transfer dissociation of doubly protonated peptide precursors. Analytical Chemistry, 79(2), 477-485. doi:10.1021/ac061457f

van den Bos, W., Cohen, M. X., Kahnt, T., \& Crone, E. A. (2012). Striatum-Medial Prefrontal Cortex Connectivity Predicts Developmental Changes in Reinforcement Learning. Cereb Cortex, 22(6), 1247-1255. doi:10.1093/cercor/bhr198

Van Overwalle, F. (2009). Social cognition and the brain: a meta-analysis. Human Brain Mapping, 30(3), 829-858. doi:10.1002/hbm.20547

Voegler, R., Peterburs, J., Bellebaum, C., \& Straube, T. (2019). Modulation of feedback processing by social context in social anxiety disorder (SAD)-an event-related potentials (ERPs) study. Scientific Reports, 9. Doi:10.1038/S41598-019-41268-0

White, S. F., Geraci, M., Lewis, E., Leshin, J., Teng, C., Averbeck, B., . . Blair, K. S. (2017). Prediction Error Representation in Individuals With Generalized Anxiety Disorder During Passive Avoidance. American Journal of Psychiatry, 174(2), 110-117. doi:10.1176/appi.ajp.2016.15111410

Winkler, A. M., Ridgway, G. R., Webster, M. A., Smith, S. M., \& Nichols, T. E. (2014). Permutation inference for the general linear model. Neuroimage, 92, 381-397. doi:10.1016/j.neuroimage.2014.01.060

Wittchen, H.-U. Strukturiertes klinisches Interview für DSM-IV: Achse I und II, SKID. 1. Aufl. edn (Hogrefe, 1997). 
Wittmann, M. K., Kolling, N., Faber, N. S., Scholl, J., Nelissen, N., \& Rushworth, M. F. (2016). SelfOther Mergence in the Frontal Cortex during Cooperation and Competition. Neuron, 91(2), 482-493. doi:10.1016/j.neuron.2016.06.022

Yan, C.G., Wang, X. Di, Zuo, X.N., Zang, Y.F., 2016. DPABI: Data Processing \& Analysis for (RestingState) Brain Imaging, Neuroinformatics. https://doi.org/10.1007/s12021-016-9299-4

Zeidman, P., Jafarian, A., Corbin, N., Seghier, M. L., Razi, A., Price, C. J., \& Friston, K. J. (2019a). A guide to group effective connectivity analysis, part 1: First level analysis with DCM for fMRI. Neuroimage, 200, 174-190. doi:10.1016/j. neuroimage.2019.06.031

Zeidman, P., Jafarian, A., Seghier, M. L., Litvak, V., Cagnan, H., Price, C. J., \& Friston, K. J. (2019b). A guide to group effective connectivity analysis, part 2: Second level analysis with PEB. Neuroimage, 200, 12-25. doi:10.1016/j.neuroimage.2019.06.032 
Reward Probability Observation o Trial t

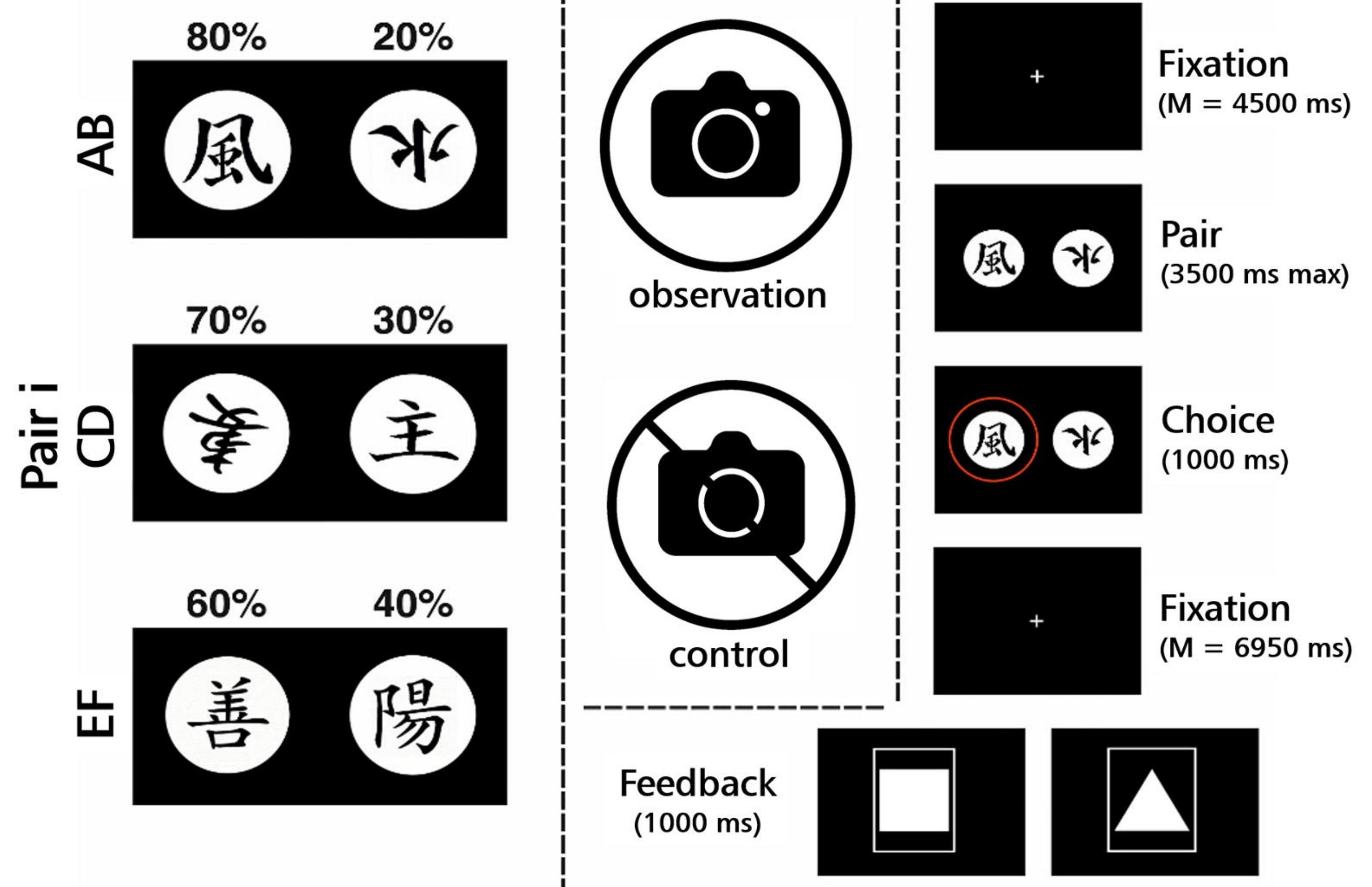




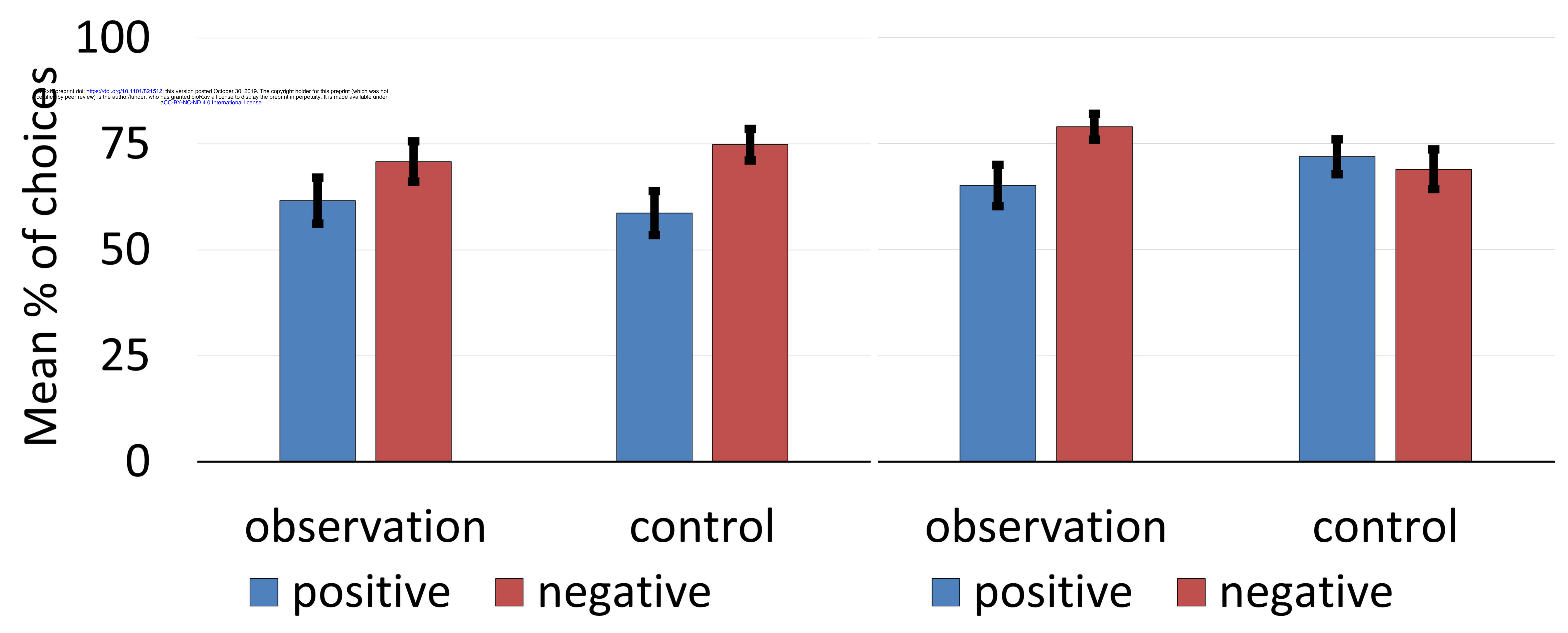




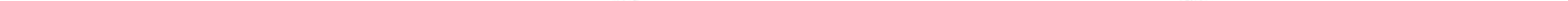



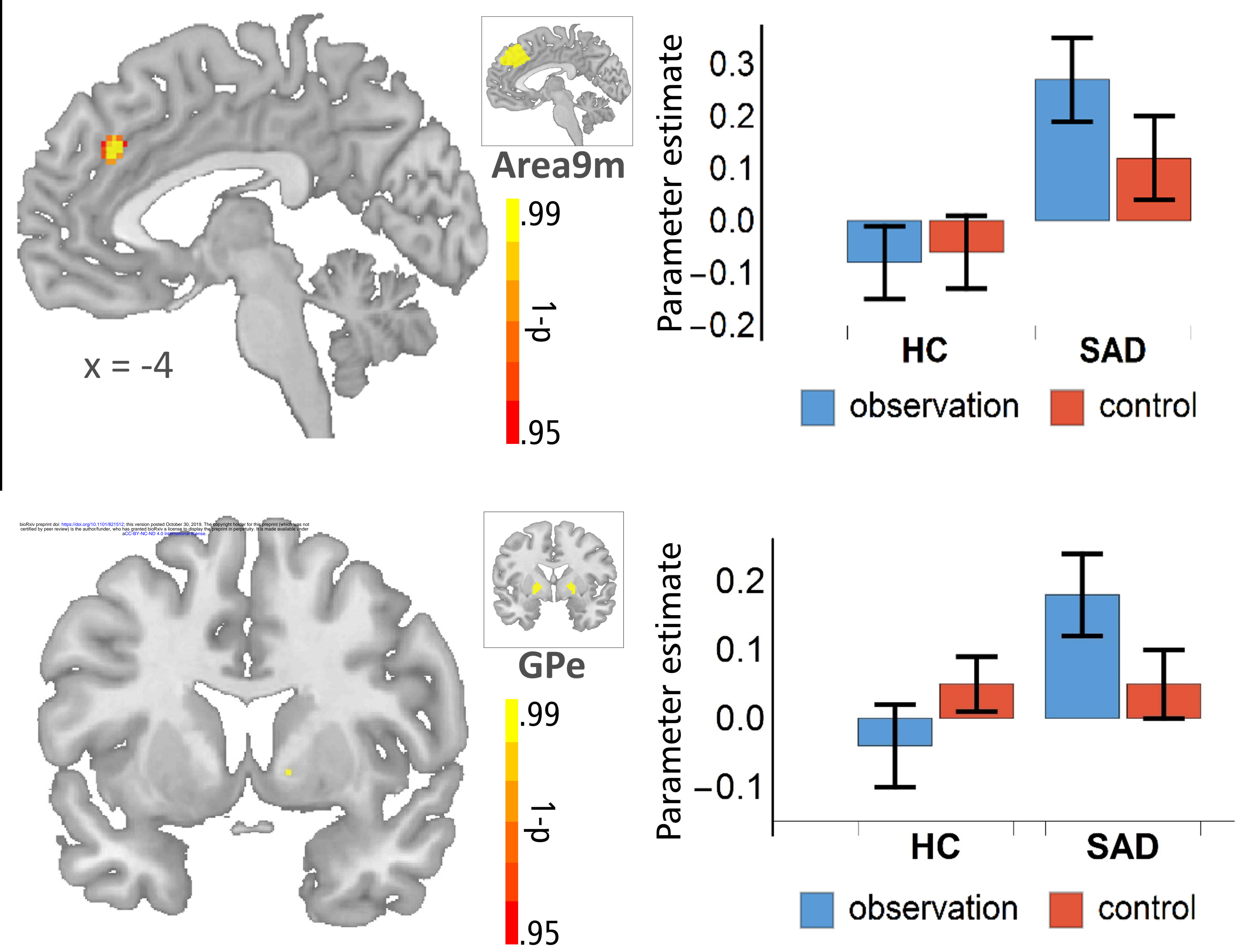

observation control 
